\title{
Asymmetric activation of number codes in bilinguals: Further evidence for the encoding complex model of number processing
}

\author{
ALLAN B. I. BERNARDO \\ De La Salle University, Manila, Philippines
}

\begin{abstract}
In two experiments, Filipino-English bilinguals were asked to verify simple addition equations that were presented either in digit, verbal-Filipino, or verbal-English formats and that included different types of sum probes. The main results show (1) faster and more accurate processing of digit and English items than of Filipino items, (2) stronger associative interference by type of probe with the digit and English items compared with the Filipino items, and (3) priming of responses from English to digit codes, and from Filipino to digit codes, but not vice versa. The results were explained by using an elaborated version of Campbell's (1994) encoding complex model with additional assumptions to address the experience of bilinguals. The additional assumptions relate to the preference among the bilingual's two verbal formats, the different strengths of activation pathways within each format, and the asymmetric activation across formats.
\end{abstract}

People encounter and process number information in various physical formats. A particular number may be perceived in its visual digit format (e.g., 7), in visual and auditory verbal formats (e.g., seven in English, sept in French, chi in Mandarin, pito in Filipino), or even in visual codes in the Roman numeral system (e.g., VII). But is the processing of number information affected by these variations in physical format? This question is of critical concern in research on numerical cognition because it directly relates to the nature of the structures and processes involved in processing number information. In particular, whether or not variations in the physical form of the number stimuli affect processing reflects either the independence or the interrelatedness of the different processes involved in numerical calculation. This paper reports the results of two experiments in which the effects of physical format on the retrieval of simple addition facts among Filipino-English bilinguals were investigated.

\section{Encoding Complex Model of Number Processing}

Among the various theories of numerical cognition, one model explicitly assumes that the processes involved

This research was supported by Grant H-141 from the Department of Science and Technology of the Republic of the Philippines, through the National Academy of Science and Technology and the National Research Council of the Philippines. Preparation of this paper was supported in part by a grant from the College of Education Research Council of De La Salle University, Manila. I thank Niño José Mateo, Christopher Cadua, Alma San Buenaventura, and Chinee Tan for their assistance in preparing the materials for the study and in gathering and encoding the data, Dr. Bienvenido Santos, Carol Santa María, and the students of Lourdes School, Quezon City, for their cooperation during the data-gathering activities, and Jamie Campbell and Ewald Neumann for their very thoughtfulcomments on an earlier version of this paper. Correspondence should be addressed to A. B. I. Bernardo, College of Education, De La Salle University, 2401 Taft Avenue, Manila 1004, Philippines (e-mail: claabb@dlsu.edu.ph). in the retrieval of arithmetic facts are largely dependent on the physical format of the number information. This model is the encoding complex model proposed by Campbell and Clark (1988). The model assumes that arithmeticfact retrieval is mediated by a variety of format-specific representations. These representations range from orthographic codes for written numerals and number words to auditory codes for spoken number words to nonverbal motor codes for digits, among others. According to Campbell (1994), the specific retrieval processes for arithmetic facts vary because each of the presentation formats may "potentially provide independent pathways to number fact representations" (p. 7). More specific assumptions for the model (from Campbell, 1994, 1995; Campbell, Kanz, \& Xue, 1999) state that: (1) number processing uses only these format-specific representations and not any abstract (format-independent) representations; (2) differences in the efficiency of number processing tasks using a specific representation code depends on the person's experience with the specific task and representation code combination; and (3) the various representation codes are associatively linked, so the activation of one representational code has the potential capacity to activate other codes.

These assumptions of the encoding complex model were proposed at least in part as an antithesis to abstractmodular theories that assume format-independent processing of number information. For example, McCloskey and colleagues (see, e.g., McCloskey \& Macaruso, 1995; McCloskey, Sokol, \& Goodman, 1986) propose that different surface formats of numbers are converted to a common abstract code and that all actual number operations are based on the abstract code. The effect of format is assumed to be limited to the process of transcoding to the abstract representation (see also, Noël, Fias, \& Brys- 
baert, 1997). Moreover, the effects of encoding are predicted to be noninteractive (i.e., additive) with the actual number processes. ${ }^{1}$

A number of studies have found format effects on number processing and interactive effects between format and number processes. For example, Campbell and Clark (1992) asked normal adult subjects to do a speeded multiplication task using problems presented either in Arabic-digit or English-verbal format. Overall, performance was poorer for the problems in English, and the decrement in performance in English was greater with more difficult problems. Campbell (1994; see also Noël et al., 1997) replicated these findings with addition and multiplication problems and also found that specific types of errors interacted with the problem format. These and other findings are inconsistent with the formatindependent assumptions of abstract-modular models of number processing, but are quite consistent with the format-specific assumptions of the encoding complex model.

\section{Encoding Complex Model and Numerical Cognition in Bilinguals}

The aforementioned propositions can be studied with bilinguals who can process number facts in at least one more format than monolinguals. The study of how bilinguals process number facts allows for more fine-tuned tests of propositions regarding the effects of format on number cognition. The results of most recent studies on number cognition among bilinguals seem to be more consistent with the encoding complex model (but see Noël \& Fias, 1998, for a contrary opinion). For example, Marsh and Maki (1976) gave addition problems in digit format to bilinguals and asked them to reply in either their first or second language. The subjects were consistently faster in their first language. McClain and Huang (1982) replicated that study and also manipulated the language of presentation. They found that the language of the items affected processing speed. Frenck-Mestre and Vaid (1993) asked English-French bilinguals to verify arithmetic facts presented in digit and first- and secondlanguage formats. They found that verification was increasingly slower and less accurate from the digit to the first- and second-language formats. More important, they found that the associative interference effects, which are generally taken as evidence for automatic activation of number facts, varied across the different formats.

Recently, Campbell et al. (1999) studied the effects of language format on different number tasks with ChineseEnglish bilinguals. The stimuli were presented in either digit or Chinese Mandarin symbols, and the subjects were asked to respond verbally in either English or Chinese. They found that arithmetic was faster when the subjects had to respond in Chinese rather than in English, and this advantage was even greater when the stimuli were in Mandarin symbols. The format effects were attributed to the use of different associative networks for number facts in the different languages and formats and to the different strength and efficiency of these associative networks that depended on prior experience of the subjects in using a specific format for a particular task.

\section{Some Proposed Elaborations on the Encoding Complex Model}

Campbell et al.'s (1999) findings and conclusions give rise to interesting possibilities. For example, they assert that the arithmetic memory system would be stronger in the language used to learn and practice basic arithmetic. If this is so, it is possible that processing of number facts would be more efficient in the bilingual's second language than in his/her first language. Such a case can arise if the bilingual uses the second language more extensively for a specific number-processing task.

Campbell et al. (1999) also assert that the arithmetic memory system in the bilingual's less preferred language exists but that this may be not as well developed. In particular, the associative links among number facts may not be as well established. They speculate that the memory system in the less preferred language would develop because results of arithmetic calculation would sometimes need to be expressed in this language. Initially, the completion of such a task is mediated by the arithmetic memory system in the preferred language. With more experience in the less preferred language, direct retrieval of the arithmetic facts in this language becomes possible. These propositions raise the question of how the different memory systems are related to each other. Is the memory system in the less preferred language more strongly linked to that of the preferred language than to that of the digit system? Does the mediation process described above necessarily involve the memory system in the preferred language? How would the memory systems of the two verbal codes be related?

On the basis of observations and specific principles in the reviewed literature, I propose a model of number processing in bilinguals that is derived from the basic assumptions of the encoding complex theory but includes specific assumptions about the relative strengths of the different memory systems in each of the formats and about the links between the different arithmetic memory systems. I refer to the model as the bilingual encoding complex model (BECM). Consistent with the encoding complex theory, the BECM assumes that bilinguals represent number facts in at least three distinct memory codes (digit codes, verbal codes in the first language, and verbal codes in the second language). The processing of arithmetic facts involves the activation and retrieval of information in one of the codes, depending on the format of the stimulus.

The BECM further proposes that the nature of the links among the different codes is shaped by such factors as preference for and prior experience with processing using each specific code (Ashcraft, 1992). Between the two verbal codes, additional factors like proficiency in the language or length of number words (Ellis \& Hen- 
nelly, 1980) might also determine preference for one verbal code over the other. Hence, among the three codes, there should be primary, secondary, and tertiary codes based on relative efficiency of processing, as determined by the aforementioned factors.

In this connection, the BECM also makes assumptions in regard to the nature of the associative links among the different number facts within each code. The same associative links and pathways are assumed to exist in each of the codes, but the strengths of these links may vary. For the strong codes, the items are strongly associated with each other (Ashcraft, 1992; Zbrodoff, 1995) so that activation of related information within such codes happens autonomously (Zbrodoff \& Logan, 1986) or automatically (LeFevre, Bisanz, \& Mrkonjic, 1988). But for the weaker codes, the different pieces of information are weakly associated with each other; hence, activation of related information is less automatic. Thus, the BECM predicts that the spreading activation effects would be evident when processing inputs use the strong codes, but absent when they use the weaker codes.

In regard to the links among the different codes, the BECM considers two factors: the strength of the associative memory network of the code and the processes involved in the development of the different codes. If the weaker codes were initially developed through the mediation of a stronger code, the BECM assumes that the activation of a weaker code would spread to the stronger code. But since the stronger codes develop independently of the weaker codes, activation of a stronger code would not necessarily spread to the weaker ones. Thus, the BECM predicts an asymmetry in the activation of number codes; activation of weaker codes would prime a relevant stronger code, but not vice versa.

To summarize, I propose two sets of assumptions in addition to the basic assumptions of the encoding complex theory. The first set refers to the nature of the links in specific number information within one code, which has implications for spreading activation effects within a code. The second set relates to the nature of the associative links among the different codes, which predicts asymmetric activations of numbers across codes of different strength.

\section{EXPERIMENT 1}

Experiment 1 tested the hypotheses regarding the relative strength and efficiency of the bilingual's three arithmetic memory codes. This experiment is a partial replication of the Frenck-Mestre and Vaid (1993) study in which an arithmetic verification task was used. ${ }^{2}$ Bilinguals were asked to mentally add two single digit numbers presented visually and then to verify whether a subsequently presented probe was the sum of the two. On certain trials, the probe was the correct sum, but on other trials the probe was a number that deviated from the correct sum by either a small or a large numerical difference. The incorrect probes that deviated by \pm 1 or 2 were the small-split probes, and those that deviated by \pm 5 or 6 were the large-splitprobes. In a block of trials, the subjects would verify three types of probes: correct sums, small-split probes, and large-split probes. The subjects were asked to work on three blocks of trials. In each block, the numbers were presented in one of three formats: digit, verbal-Filipino, or verbal-English.

Several hypotheses were posed. (1) Verification times should vary as a function of presentation format (FrenckMestre \& Vaid, 1993). (2) Verification times should be fastest with the digit format (LeFevre, Bisanz, \& Mrkonjic, 1988). (3) Because the subjects preferred to do mental addition in English (their second language) rather than in Filipino (their first language), verification times should be faster for English than for Filipino items. (4) Verification times for the correct sums should be faster than for incorrect probes. (5) As has been shown in previous studies (e.g., Ashcraft \& Stazyk, 1981) and is consistent with the assumptions of automatic spreading activation of number facts, verification of small-split probes should be longer than that of large-split probes in the digit and verbal-English formats. (6) As the associative links within the weaker code are not yet well established, the interference related to the small-split probe should not be found in the verbal-Filipino format (Frenck-Mestre \& Vaid, 1993).

\section{Method}

Subjects. Twenty-one high school students participated as part of a class requirement. They were all bilingual in Filipino and English, with Filipino as their first language. All were studying in a bilingual education program and had been trained in mathematics with English as the medium of instruction (i.e., English language textbooks, learning materials, and examinations since their first year of formal schooling). All reported that they preferred to do mental arithmetic in English. The subjects participated in groups of 10 or 11 subjects per session.

Stimuli. A problem trial consisted of two numbers (or addends) separated by a plus sign (e.g., $2+3$ ), followed by a probe (e.g., 5). The addends were always single digits from 1 to 9 ; the probes were from 2 to 10 . In one block of trials, there were 16 problem trials grouped into four types: (1) four buffer trials, (2) four trials with correct sums, (3) four trials with small-split probes, and (4) four trials with large-split probes. The data from buffer problems were not analyzed but the items were used for two reasons. First, for each block of trials two buffer items were always in the first and second trial as a precaution against subjects' hesitating at the start of a block. Second, the probe for the buffer items was always the correct sum of the addends so that there were an equal number of positive and negative responses within a block.

The 16 problems were different across the four types. But the same 16 problems were presented in three formats: digit, verbalFilipino, and verbal-English. The presentation of trials in one format was blocked. The order of presentation of the three formats was counterbalanced using a Latin-square design, which ensured that across all subjects there was an equal probability that a subject would do any of the three formats first, second, or third.

Procedure. The problems were presented on a personal computer. The problems were in black, 5-mm uppercase characters against a white screen. Each trial began with the appearance of a fixation point at the center of the screen. The subjects were told to focus on this point. Two addends appeared on the location of the fixation point. There was a "+" between the addends, and no space sepa- 
Table 1

Mean Response Times (RT, in Seconds) and Standard Errors $(S E)$ as a Function of Format and Probe Type in Experiment 1

\begin{tabular}{|c|c|c|c|c|c|c|}
\hline \multirow[b]{3}{*}{ Probe Type } & \multicolumn{6}{|c|}{ Format of Presentation } \\
\hline & \multicolumn{2}{|c|}{ Digit } & \multicolumn{2}{|c|}{ Verbal-English } & \multicolumn{2}{|c|}{ Verbal-Filipino } \\
\hline & RT & $S E$ & RT & $S E$ & RT & $S E$ \\
\hline Sum & 0.780 & .064 & 1.190 & .082 & 1.805 & .153 \\
\hline Large split & 0.986 & .095 & 1.471 & .183 & 1.898 & .232 \\
\hline Small split & 1.145 & .104 & 1.827 & .182 & 1.923 & .166 \\
\hline
\end{tabular}

rated these figures. The addends were presented for $250 \mathrm{msec}$, after which they were masked by asterisks for $50 \mathrm{msec}$, and then replaced by the probe number. The probe stayed on the screen until the subject gave an answer by pressing the appropriate keys marked " $\mathrm{T}$ " for true and "F" for false. (The assignment of $T$ and $F$ keys to the left and right hands was varied randomly across subjects.) No feedback was given. The next trial began $2 \mathrm{sec}$ after the subject responded.

To acquaint the subjects with the task, they were given a practice set of 10 trials in the different formats. For the actual trials, the subjects completed three sets of 16 problems; each set was separated by a $30-$ sec rest period.

\section{Results and Discussion}

Response times. The mean response times for the correct sum, small-split, and large-split items for each of the three formats were computed and are summarized in Table 1. Times for error trials (i.e., those trials in which the subjects answered incorrectly) were not included in the analysis. The means were submitted to a 3 (format) $\times 3$ (probe type) repeated-measures analysis of variance (ANOVA). As predicted, there was a main effect of presentation format $[F(2,40)=38.07, p<.0001]$. The subjects were faster with the digit format $(0.970 \mathrm{sec})$, followed by the verbal-English $(1.115 \mathrm{sec})$ and verbal-Filipino $(1.875 \mathrm{sec})$ format. Also as predicted, there was a main effect of probe type $[F(2,40)=11.17, p<.0001]$. The subjects were fastest for the correct sum $(1.259 \mathrm{sec})$ and the large-split probes $(1.467 \mathrm{sec})$ and slowest with the small-split probes $(1.632 \mathrm{sec})$.

There was also an interaction between format and probe type $[F(4,80)=2.52, p<.05]$. This interaction was further explored by conducting independent analyses on each of the presentation formats to determine the specific effects of probe type within each format. For digitformat items, there was a main effect of probe type $[F(2,40)=11.27, p<.0001]$. Orthogonal contrasts of means showed that the response times for correct sums were faster than for large-split probes $[F(2,40)=6.08$, $p<.03]$ and that times for large-split probes were faster than for small-split probes $[F(2,40)=4.39, p<.05]$. The pattern was similar for items presented in verbal-English format. The main effect of probe was significant $[F(2,40)=$ $12.34, p<.0001]$. The subjects were faster with correct sums than with large-split probes $[F(2,40)=6.08, p<$ $.03]$ and faster with large-split probes than with smallsplit probes $[F(2,40)=4.39, p<.05]$. There was no significant main effect of probe $(F<1)$ for items in the verbal-Filipino format.
As predicted, the response time pattern associated with an automatic spreading activation network for number facts was found with the digit and verbal-English formats but not with the verbal-Filipino formats. With the digit and English formats, the subjects were faster in their processing of the correct sums, presumably because the answers could be directly compared to a well-developed memory representation of number facts in the two formats (Campbell \& Tarling, 1996). This effect was not found with the Filipino format, which suggests that such well-developed representations may not yet exist for the Filipino code.

Error rates. The error rates are summarized in Table 2 and were analyzed with the same ANOVA. There was a main effect presentation format $[F(2,40)=6.66, p<$ $.004]$. Errors were lowest for the digit format (.08), followed by the verbal-English (.13) and verbal-Filipino (.21) formats. The high error rates with the verbal-Filipino items complement the slow response times and indicate that the subjects had difficulty with these items. There was a main effect of probe type $[F(2,40)=4.63, p<.02]$. The subjects made the fewest errors for the large-split (.10) and sum (.12) probes and the most with small-split probes (.19), consistent with the slower processing times with the same probe type. There was also no interaction between format and probe type $\left[F(4,80)=1.33, M S_{\mathrm{e}}=\right.$ $0.02, p>.10]$. Overall, the main effects for the error rates are consistent with the response times and do not indicate any speed-accuracy trade-off.

Some inferences can be drawn from the results so far. First, consistent with previous research, processing of number facts was most efficient for the digit format. Second, processing was also efficient for the verbalEnglish items but not for the verbal-Filipino items, which verifies the subjects' reports that they perform mental addition in English and not in Filipino. The results seem contrary to previous research that has shown that better processing occurs in bilinguals' first language, but are consistent with the assumptions of the BECM. Third, error-split response times show associative interference for digit and verbal-English formats, suggesting that the memory representations for the two formats are in the form of associative networks. Finally for the verbal-Filipino items, there was no difference in the processing times for true and false items and no associative interference since the split effect was absent. These results together with the slow processing times and high error rates for the same

Table 2

Mean Error Rates and Standard Errors $(S E)$ as a Function of Format and Probe Type in Experiment 1

Format of Presentation

\begin{tabular}{|c|c|c|c|c|c|c|}
\hline \multirow[b]{3}{*}{ Probe Type } & \multicolumn{6}{|c|}{ Format of Presentation } \\
\hline & \multicolumn{2}{|c|}{ Digit } & \multicolumn{2}{|c|}{ Verbal-English } & \multicolumn{2}{|c|}{ Verbal-Filipino } \\
\hline & Error & $S E$ & Error & $S E$ & Error & $S E$ \\
\hline Sum & .05 & .02 & .10 & .03 & .23 & .05 \\
\hline Large split & .07 & .04 & .07 & .03 & .17 & .05 \\
\hline Small split & .11 & .03 & .21 & .06 & .25 & .07 \\
\hline
\end{tabular}


items suggest that the underlying representation for verbalFilipino items does not consist of strongly associated codes.

In summary, the processing of number facts in both digit and verbal-English formats shares important characteristics - in both cases, processing is fast, relatively few errors occur, and both show associative interference. This is not the case with the processing of number facts in verbal-Filipino format. The general pattern of results mirrors the pertinent findings of Frenck-Mestre and Vaid (1993) except that the preferred verbal format of the subjects is in their second language rather than their first.

\section{EXPERIMENT 2}

Experiment 2 was designed to explore the relationship between the digit and verbal codes in bilinguals' memory representation of number facts. The same methodology used earlier was employed but with two important changes. First, the format of the addends was manipulated between groups; thus, for some subjects, all addends were presented in digit format, for others in verbalFilipino, and so on. Second, all subjects were presented probes in all three formats. Therefore, all subjects experienced trials in which the addends and probes were in the same format and trials in which the two sets of stimuli were in different formats.

By manipulating the code of the addends and the code of sum, we were able to test for whether the activation of one code consequently spreads to the others. For example, the activation of the primary code (digit) is predicted not to spread to the two weaker codes because the primary code developed independently and is often used independently of the weaker codes. Thus, when digit addends are given, the subjects should be faster when the sum is also a digit since this code is already activated, but they should be equally slow with the two other codes since the activation of the digit code does not prime the others. On the other hand, activation of the secondary code (verbal-English) should spread to the stronger digit code because the verbal-English code developed with reference to the digit code. Thus, the subjects should be just as fast with digit as with verbal-English sums. Since the verbal-English code is not expected to prime the verbalFilipino code, the subjects should be slower in responding to Filipino sums. The prediction for Filipino addends is less straightforward. Activation of the verbal-Filipino code is expected to prime the stronger code that mediated the development of the weaker code. Campbell et al. (1999) suggest that this mediating code is the stronger verbal code. But this might not be so for the bilinguals in the present study; there is as yet no evidence that either or both the digit or verbal-English codes mediate the development of verbal-Filipino codes. The activation of the verbal-Filipino code could prime one or both of the stronger codes, depending on what code mediates the Filipino code. Thus, it was predicted that the subjects would respond faster to the stronger code(s) that medi- ated the development of the Filipino code, whatever that code(s) might be.

The other set of hypotheses relates to the automatic activation of number of facts within each code. The interference created by incorrect sums (i.e., small-split probes) is expected to interact with format of the probe, but not with format of the addends, because it is assumed that it is within the code activated by the probe that the interference effects will operate. As with Experiment 1, interference due to the small-split probes was expected to be stronger for the digit and verbal-English probes and weaker for the verbal-Filipino probes.

\section{Method}

Subjects. Fifty-nine high school students similar in profile to those in Experiment 1 participated in this study. Each student was assigned randomly to one of the three addend-format conditions.

Stimuli and Procedures. Similar stimuli, procedures, and control features to those in Experiment 1 were used. But, in Experiment 1 , the format of the addends was different for each set of trials, whereas in Experiment 2, the format of the addends was the same for all sets of trails, and the probe was in a different format for each of the three sets.

\section{Results and Discussion}

Response times. The response times were encoded as they were in Experiment 1 and are summarized in Table 3. The means were submitted to a $3 \times 3 \times 3$ ANOVA for mixed design, with the addends format as between-groups factor, and probe format and probe type as within-groups factors. The ANOVA showed a main effect of format of addends $[F(1,57)=75.29, p<.0001]$. The subjects working with the digit-format addends were fastest $(1.474 \mathrm{sec})$, followed by those working with the verbal-English $(1.635 \mathrm{sec})$ and verbal-Filipino $(1.748 \mathrm{sec})$ addends.

There was no main effect of format of probe $(F<1)$. Consistent with the predictions, there seemed to be an interaction between format of addends and format of probe but the effect was only marginally significant

Table 3

Mean Response Times (RT, in Seconds) and Standard Errors $(S E)$ as a Function of Format of Addends, Format of Probe, and Probe Type in Experiment 2

\begin{tabular}{|c|c|c|c|c|c|c|}
\hline \multirow[b]{3}{*}{ Probe Type } & \multicolumn{6}{|c|}{ Format of Probe } \\
\hline & \multicolumn{2}{|c|}{ Digit } & \multicolumn{2}{|c|}{ Verbal-English } & \multicolumn{2}{|c|}{ Verbal-Filipino } \\
\hline & RT & $S E$ & RT & $S E$ & RT & $S E$ \\
\hline \multicolumn{7}{|c|}{ Digit Addends } \\
\hline Sum & 0.746 & .064 & 0.887 & .085 & 0.923 & .056 \\
\hline Large split & 0.891 & .091 & 0.865 & .085 & 0.997 & .073 \\
\hline Small split & 0.968 & .076 & 1.094 & .143 & 0.985 & .068 \\
\hline \multicolumn{7}{|c|}{ Verbal-English Addends } \\
\hline Sum & 1.404 & .149 & 1.474 & .132 & 1.850 & .158 \\
\hline Large split & 1.625 & .147 & 1.050 & .154 & 1.825 & .220 \\
\hline Small split & 1.801 & .167 & 1.935 & .160 & 1.910 & .176 \\
\hline \multicolumn{7}{|c|}{ Verbal-Filipino Addends } \\
\hline Sum & 1.667 & .107 & 1.971 & .141 & 2.370 & .295 \\
\hline Large split & 2.032 & .148 & 2.343 & .168 & 2.265 & 177 \\
\hline Small split & 2.202 & .139 & 2.668 & .192 & 2.708 & .241 \\
\hline
\end{tabular}


$[F(2,114)=2.45, p=.09]$. It is possible that the interaction effect was only marginally significant because of the variance contributed by the trials requiring negative responses, so a partial analysis of the interaction between the format of the addends and the format of the probes was conducted on the response time data for the correct sum trials. The interaction effect was found to be statistically significant $[F(2,114)=3.06, p=.05]$.

The interaction between format of addends and format of probe was further explored by doing appropriate orthogonal analyses with both the complete data set and the correct sum subset. The analyses showed that the specific effects of probe format on each format of addends was consistent with the predictions and was true for both the complete data set and the correct sum subset. However, for the complete data set, some of the effects were only marginally significant. The simple effects and orthogonal comparisons are summarized in Table 4.

The results regarding the interaction between the format of the addend and the format of the probe verify the hypotheses related to the asymmetric activation of number codes. It does seem that stronger codes do not prime weaker codes, but that weaker codes prime the stronger code that figured in the former's development. With the digit addends, the subjects were fastest with digit probes but were equally slow with the two verbal probes, since the two verbal codes were not primed by the primary digit code. With the verbal-English addends, the subjects were equally fast with the digit and verbal-English probes since both codes are activated. But they were slower with Filipino probes since this code was not primed. Response times for Filipino addends were somewhat problematic. The subjects were faster with the digit probe than with verbal-English probe, suggesting the Filipino addends primed the digit codes rather than the latter. But the subjects were also quite slow with the Filipino probes, although it was expected that they would be faster with this already activated code. That the Filipino addendFilipino probe stimuli were slow could indicate that the subjects were still referring to the primary code, consistent with Campbell et al.'s (1999) proposal that the preferred code mediates the processing of arithmetic facts in the weaker code.

The results related to the effects of probe type verify the other hypotheses regarding the activation of number stimuli within different formats. There was no overall main effect of probe type $(F<1)$, but, as predicted, there was an interaction between probe type and format of addends $[F(2,114)=4.45, p<.02]$. (None of the other interaction effects were statistically significant.) The interaction effect was further studied by looking at the simple effects of probe type on each format of addends. For the subjects who were presented addends in digit format, the effect of probe type was significant $[F(2,38)=12.82$, $p<.0001]$. The subjects were faster with correct sums $(0.852 \mathrm{sec})$ than with large-split probes $(0.918 \mathrm{sec})$ $[F(1,19)=14.13, p<.002]$. They were also faster with large-split $(0.918 \mathrm{sec})$ than with small-split probes $(1.016 \mathrm{sec})[F(1,19)=6.61, p<.02]$. For the subjects who were presented addends in verbal-English format, there was also an effect of probe type $[F(2,38)=4.59$, $p<.02]$. The small-split probes $(1.883 \mathrm{sec})$ were again the slowest, the time was slower than for large-split probes $(1.658 \mathrm{sec})[F(1,19)=5.67, p<.03]$, but there was no difference in response times for sum probes $(1.576 \mathrm{sec})$ or large-split probes $(1.658 \mathrm{sec})$. For the subjects who were presented addends in the verbal-Filipino format, the effect of probe type was significant $[F(2,36)=$ $13.05, p<.0001]$. Times for correct sums $(2.003 \mathrm{sec})$ were faster than for large-split probes $(2.213 \mathrm{sec})[F(1,18)=$ $4.31, p=.05]$, which were faster than the small-split probes $(2.526 \mathrm{sec})[F(1,18)=10.84, p<.005]$.

The results related to the interaction of interference effects of the probe type with format of addends mostly replicate those in Experiment 1. Again, there were inter-

Table 4

Simple Effects and Orthogonal Comparisons for Addend Format $\times$ Probe Format Interaction

\begin{tabular}{|c|c|c|c|c|}
\hline & \multicolumn{2}{|c|}{ Complete Data } & \multicolumn{2}{|c|}{ Correct Sums } \\
\hline & Means & $F$ Scores & Means & $F$ Scores \\
\hline \multicolumn{5}{|c|}{ Digit Addends } \\
\hline Simple effect of probe format & & $F(2,38)=2.61, p=.087$ & & $F(2,38)=6.35, p<.005$ \\
\hline Digit vs. English & 0.868 vs. 0.966 & $F(1,19)=3.29, p=.085$ & 0.746 vs. 0.887 & $F(1,19)=6.53, p<.02$ \\
\hline Digit vs. Filipino & 0.868 vs. 0.968 & $F(1,19)=6.60, p=.02$ & 0.746 vs. 0.923 & $F(1,19)=18.29, p<.0004$ \\
\hline English vs. Filipino & 0.966 vs. 0.968 & $F(1,19)<1.0$ & 0.887 vs. 0.923 & $F(1,19)<1.0$ \\
\hline \multicolumn{5}{|c|}{ English Addends } \\
\hline Simple effect of probe format & & $F(2,38)=3.18, p=.05$ & & $F(2,38)=7.42, p<.002$ \\
\hline Digit vs. English & 1.610 vs. 1.645 & $F(1,19)<1.0$ & 1.404 vs. 1.474 & $F(1,19)<1.0$ \\
\hline Digit vs. Filipino & 1.610 vs. 1.862 & $F(1,19)=5.56, p<.03$ & 1.404 vs. 1.850 & $F(1,19)=12.92, p<.002$ \\
\hline English vs. Filipino & 1.645 vs. 1.862 & $F(1,19)<3.97, p=.06$ & 1.474 vs. 1.850 & $F(1,19)=11.12, p<.004$ \\
\hline \multicolumn{5}{|c|}{ Filipino Addends } \\
\hline Simple effect of probe format & & $F(2,36)=3.76, p<.04$ & & $F(2,36)=4.42, p<.02$ \\
\hline Digit vs. English & 1.967 vs. 2.328 & $F(1,18)=11.32, p<.02$ & 1.669 vs. 1.971 & $F(1,18)=5.85, p<.03$ \\
\hline Digit vs. Filipino & 1.967 vs. 2.447 & $F(1,18)=4.65, p<.05$ & 1.669 vs. 2.370 & $F(1,18)=6.09, p<.03$ \\
\hline English vs. Filipino & 2.328 vs. 2.447 & $F(1,18)<1.0$ & 1.971 vs. 2.370 & $F(1,18)=2.23, p>.10$ \\
\hline
\end{tabular}


ference effects with digit and verbal-English probes, but the interference effects were also demonstrated with the verbal-Filipino probes, unlike those in Experiment 1. The interference effects found with verbal-Filipino probes could suggest automatic activation of number facts in this code, which is inconsistent with the hypothesis and the results of Experiment 1. The inconsistency could be due to the difference in design between the two experiments. In Experiment 1, the subjects who processed Filipino stimuli did so in a block of Filipino stimuli trials. They did not have to process stimuli using English or digit items, nor were they required to process mixed code stimuli. In Experiment 2, the subjects processed pure Filipino and mixed code stimuli. It is conceivable that this setup might have encouraged the subjects to mentally transcode the stimuli into the stronger code in Experiment 2. If so, the subjects could have used the stronger code to mediate number processing, thus producing the interference effects not found earlier.

Error rates. The mean error rates are summarized in Table 5 and were analyzed with the same ANOVA procedures. The ANOVA revealed no significant main effect for any of the independent variables and only one interaction effect was significant. There was an interaction between probe format and probe type $[F(4,224)=$ $2.54, p<.05]$. In order to understand this interaction effect better, the simple effects and appropriate comparison analysis were conducted. The simple effect of probe type on the digit probes was significant $[F(2,116)=3.34$, $p<.04]$. The subjects were more accurate with sum probes than with large-split probes $[.08$ vs. . $14 ; F(1,58)=$ $7.81, p<.01]$, but none of the other pairwise comparisons revealed significant differences. There was also a significant simple effect of probe type on verbal-English probes $[F(2,116)=3.00, p=.05]$. The subjects made more errors with small-split probes than with large-split probes $[.16$ vs. $.08 ; F(1,58)=12.82, p<.01]$; no other compari-

Table 5

Mean Error Rates and Standard Error ( $S E$ ) as a Function of Format of Addends, Format of Probe, and Probe Type in Experiment 2

\begin{tabular}{|c|c|c|c|c|c|c|}
\hline \multirow[b]{3}{*}{ Probe Type } & \multicolumn{6}{|c|}{ Format of Probe } \\
\hline & \multicolumn{2}{|c|}{ Digit } & \multicolumn{2}{|c|}{ Verbal-English } & \multicolumn{2}{|c|}{ Verbal-Filipino } \\
\hline & Error & $S E$ & Error & $S E$ & Error & $S E$ \\
\hline \multicolumn{7}{|c|}{ Digit Addends } \\
\hline Sum & .10 & .03 & .13 & .04 & .15 & .06 \\
\hline Large split & .15 & .04 & .11 & .03 & .06 & .03 \\
\hline Small split & .09 & .03 & .15 & .04 & .09 & .03 \\
\hline \multicolumn{7}{|c|}{ Verbal-English Addends } \\
\hline Sum & .06 & .03 & .22 & .05 & .16 & .05 \\
\hline Large split & .11 & .04 & .22 & .05 & .15 & .05 \\
\hline Small split & .11 & .04 & .24 & .05 & .15 & .05 \\
\hline \multicolumn{7}{|c|}{ Verbal-Filipino Addends } \\
\hline Sum & .07 & .03 & .11 & .04 & .13 & .04 \\
\hline Large split & .15 & .05 & .05 & .02 & .18 & .06 \\
\hline Small split & .15 & .04 & .22 & .07 & .14 & .06 \\
\hline
\end{tabular}

son revealed significant differences. There was no simple effect of probe type with verbal-Filipino probes $(F<1)$. Overall, the pattern of errors does not point to any speedaccuracy trade-off related to the verification times or to any result that is contrary to the reaction times findings.

The results of Experiment 2 verify important propositions of the BECM. First, the results replicate the decreasing efficiency of processing from digit to verbalEnglish to verbal-Filipino found in Experiment 1. The results also verify the propositions regarding the links across the different format codes. Finally, the results show that the associative pathways are similar across the different format codes, although the pathways seem to be weaker with the verbal-Filipino code.

\section{GENERAL DISCUSSION}

Two experiments were conducted to verify propositions of a bilingual encoding complex model for verification of number facts. The results verify the general assumptions derived from the encoding complex model and the more specific propositions of the BECM that were intended to apply to bilinguals. In particular, the results provided support for the following propositions:

1. There exist at least three codes for the bilingual, and these differ in strength and efficiency as determined by prior experience with the code.

2 . The stronger verbal code is not always in the bilingual's first language, instead it is the language used for learning and practicing arithmetic tasks.

3 . The strength of the associative pathways within each code varies across the three codes, again depending on the prior experience with the codes.

4. The activation across codes is asymmetric; weaker codes activate the relevant stronger code, but stronger codes do not activate weaker ones.

These propositions relate to format-related effects on the verification of arithmetic number facts. Format effects may differ for arithmetic production tasks and for other numerical tasks like counting or multidigit calculation, since different processes are involved in the tasks (see, e.g., Zbrodoff \& Logan, 1990). Therefore, the BECM is best viewed as a model for arithmetic verification and not as a general all-purpose model for arithmetic performance for bilinguals.

The results of the study provide new theoretical assumptions that either elaborate on or modify those reviewed earlier, particularly those proposed by Campbell et al. (1999) regarding number processing among bilinguals. For example, the Filipino-English bilinguals preferred to process numbers in English and have had more extensive experience with this task using the English verbal code, even if English is their second language. Thus, consistent with Campbell et al.'s (1999) assertion about the role of prior experience in the specific type of task (see also LeFevre \& Liu, 1997), bilingual subjects' preferred and more efficient verbal code need not always be in their native language. 
The specific nature of the number representations underlying the processing of verbal-Filipino number stimuli is still not clear. For example, two results suggest weak associative links within the Filipino code: the absence of interference effects of probe type in Experiment 1, and the absence of priming between Filipino addends and Filipino probes. Yet, the interference effect of probe type was found with Filipino addends in Experiment 2. But this result may stem from the different experimental design, which might have encouraged the subjects to transcode the stimuli into stronger codes that have stronger associative links. Thus, although most of the data patterns suggest the absence of strong associative links within the Filipino number code, this proposition needs to be studied further.

Another important insight derived from this study is about how the different codes relate to each other. This issue has not been extensively addressed in previous studies, although Campbell et al. (1999) suggest that the preferred verbal code mediates number processing in the weaker verbal code when the bilingual is still learning the number operations in this weaker code. The present findings suggest that these mediation processes might contribute to the development of pathways between the weaker code and the stronger code that mediated in the weaker code's development. In particular, the weaker codes would have associative links to the stronger code. But this link does not seem to be bidirectional; instead, it seems to be triggered from the weaker code to the stronger one, and not vice versa. The assumptions relating to the asymmetric activation of number codes is an elaboration of Campbell et al.'s (1999) proposal that needs to be further studied.

Interestingly, data from Experiment 2 suggest that what mediates the processing of the Filipino stimuli was the digit code and not the verbal-English code. But given the novel nature of the task and the lack of precise information on the strategies that the subjects were using, it seems premature to rule out the possibility that the verbalEnglish code could also mediate the processing of Filipino stimuli. This matter can be studied further by using methods that tap into the development of Filipino number codes.

The present results suggest that there are common principles that underlie processing of number facts in bilinguals and monolinguals. However, the specific factors that shape the precise features of the representation and processing of number facts among bilinguals are probably more complex. The present results suggest that the underlying number verification processes among bilinguals implicate factors related to amount of experience with number processing using a particular format and to how one format may be linked to the development of number processing in another format. Conducting similar types of studies will not only lead to a better understanding of how bilinguals think, it should help us better understand how the human mind comes to effectively process different pieces and forms of stimuli that carry the same functional units of information.

\section{REFERENCES}

Ashcraft, M. H. (1992). Cognitive arithmetic: A review of data and theory. Cognition, 44, 75-106.

Ashcraft, M. H., \& STAZYK, E. H. (1981). Mental addition: A test of three verification models. Memory \& Cognition, 9, 185-196.

Campbell, J. I. D. (1994). Architectures for numerical cognition. Cognition, 53, 1-44.

CAmpbell, J. I. D. (1995). Mechanisms of simple addition and multiplication: A modified network-interference theory and simulation. Mathematical Cognition, 1, 121-164.

Campbell, J. I. D., \& Clark, J. M. (1988). An encoding-complex view of cognitive number processing: Comment on McCloskey, Sokol, and Goodman (1986). Journal of Experimental Psychology: General, 117, 204-214.

Campbell, J. I. D., \& Clark, J. M. (1992). Numerical cognition: An encoding-complex perspective. In J. I. D. Campbell (Ed.), The nature and origins of mathematical skills (pp. 457-491). Amsterdam: Elsevier.

Campbell, J. I. D., Kanz, C. L., \& Xue, Q. (1999). Number processing in Chinese-English bilinguals. Mathematical Cognition, 5, 1-39.

CAMPbell, J. I. D., \& TARling, D. P. M. (1996). Retrieval processes in arithmetic production and verification. Memory \& Cognition, 24, 156-172.

Dehaene, S. (1992). Varieties of numerical abilities. Cognition, 44, 1-42.

Ellis, N. C., \& Hennelly, R. A. (1980). A bilingual word-length effect: Implications for intelligence testing and the relative ease of mental calculation in Welsh and English. British Journal of Psychology, 71, 43-51.

FrenCK-Mestre, C., \& VAID, J. (1993). Activation of number facts in bilinguals. Memory \& Cognition, 21, 809-818.

LeFevre, J.-A., Bisanz, J., \& Mrkonjic, L. (1988). Cognitive arithmetic: Evidence for obligatory activation of arithmetic facts. Memory \& Cognition, 16, 45-53.

LeFevre, J.-A., \& LiU, J. (1997). The role of experience in numerical skill: Multiplication performance in adults from China and Canada. Mathematical Cognition, 3, 31-62.

Marsh, L. G., \& MAKI, R. H. (1976). Efficiency of arithmetic operations in bilinguals as a function of language. Memory \& Cognition, 4, 459-464

McClain, L., \& Huang, J. Y. S. (1982). Speed of simple arithmetic in bilinguals. Memory \& Cognition, 10, 591-596.

McCloskey, M., \& Macaruso, P. (1995). Representing and using numerical information. American Psychologist, 50, 351-363.

McCloskey, M., Sokol, S. M., \& Goodman, R. A. (1986). Cognitive processes in verbal-number production: Inferences from the performance of brain-damaged subjects. Journal of Experimental Psychology: General, 115, 307-330.

NoËL, M.-P., \& FIAS, W. (1998). Bilingualism and numeric cognition. Psychologica Belgica, 38, 231-250.

NoËL, M.-P., Fias, W., \& Brysbaert, M. (1997). About the influence of the presentation format on arithmetic-fact retrieval processes. Cognition, 63, 335-374.

ZBRodoff, N. J. (1995). Why is $9+7$ harder than $2+3$ ? Strength and interference as explanations of the problem-size effect. Memory \& Cognition, 23, 689-700.

Zвrodoff, N. J., \& Logan, G. D. (1986). On the autonomy of mental processes: A case study of arithmetic. Journal of Experimental Psychology: General, 115, 118-130.

ZBrodoff, N. J., \& Logan, G. D. (1990). On the relation between production and verification tasks in the psychology of simple arithmetic. Journal of Experimental Psychology: Learning, Memory, \& Cognition, 16, 83-97.

\section{NOTES}

1. Another major theory of number processing is Dehaene's (1992) triple code model. The triple code model specifies the existence of three different internal codes for representing number information: an auditory visual code, a visual digit code, and an analog magnitude code. The different codes are used for specific numerical operation (e.g., the verbal code is used for arithmetic fact retrieval). Like the encoding complex theory, the triple code model assumed multiple codes, but like the 
abstract modular theory, a specific arithmetic operation can only be executed using one code. In regard to the effects of presentation format, the triple code model makes the same predictions as McCloskey's theory. If the input is not in the appropriate code, it will have to be transcoded before processing can be completed. Thus, the triple code model can be considered to be an exemplar of the abstract modular theories, particularly in regard to the predictions regarding the absence of format-specific effects number processing.

2. Experiment 1 differed from Frenck-Mestre and Vaid's (1993) study in two ways. First, the stimulus onset asynchrony was manipulated in their study but not in the present one. Second, the subjects in the present study all reported that they added numbers in their second language instead of in their first; in contrast, the subjects in their study added numbers in their first language.

(Manuscript received September 27, 2000; revision accepted for publication May 28, 2001.) 